\title{
Higher Order Sliding Mode Observer-Based Sensor Fault Detection in DC Microgrid's Buck Converter
}

\author{
Daijiry Narzary and Kalyana C. Veluvolu *(D) \\ School of Electronics Engineering, Kyungpook National University, Daegu 41566, Korea; narzary_daijy@knu.ac.kr \\ * Correspondence: veluvolu@ee.knu.ac.kr
}

check for updates

Citation: Narzary, D.; Veluvolu, K.C. Higher Order Sliding Mode Observer-Based Sensor Fault Detection in DC Microgrid's Buck Converter. Energies 2021, 14, 1586. https://doi.org/10.3390/en14061586

Academic Editor: Mehdi

Hosseinzadeh

Received: 31 January 2021

Accepted: 5 March 2021

Published: 12 March 2021

Publisher's Note: MDPI stays neutral with regard to jurisdictional claims in published maps and institutional affiliations.

Copyright: (c) 2021 by the authors. Licensee MDPI, Basel, Switzerland. This article is an open access article distributed under the terms and conditions of the Creative Commons Attribution (CC BY) license (https:// creativecommons.org/licenses/by/ $4.0 /)$.

\begin{abstract}
Fault detection in a Direct Current (DC) microgrid with multiple interconnections of distributed generation units (DGUs) is an interesting topic of research. The occurrence of any sensor fault in the DC microgrid should be detected immediately by the fault detection network to achieve an overall stable performance of the system. This work focuses on sensor fault diagnosis of voltage and current sensors in interconnected DGUs of the microgrid. Two separate higher order sliding mode observers (HOSM) based on model dynamics are designed to estimate the voltage and current and generate the residuals for detecting the faulty sensors in DGUs. Multiplicative single and multiple sensor faults are considered in voltage and current sensors. By appropriate selection of threshold, single and multiple sensor fault detection strategies are formulated. A hierarchical controller is designed to ensure equal sharing of current among the DGUs of the DC microgrid and stabilize the system. Simulations are performed to validate the proposed approach for various configurations of the DC microgrid under various load and off noise conditions.
\end{abstract}

Keywords: DC microgrid; distribution generation units; fault detection; higher order sliding mode observer; Lyapunov's stability; multi sensor faults

\section{Introduction}

The increase of energy demand and the concern about the climate change has led to extensive research for renewable energy sources (RES). Hence, microgrids are used to integrate different RES and electrify the remote areas continuously. A microgrid is a small scale power system that consists of loads, distribution generation units (DGUs) and other units for storage which is interconnected with power lines of the grid [1]. In a DC microgrid, a Buck converter contributes an important role for the efficient performance of the DC microgrid. The main advantage of using DC microgrid over AC microgrid is that it can be operated in grid connected or in islanded mode. DC microgrids [2-4] are more in demand than AC microgrids due to its attractive features like availability of converters, interfacing of batteries and DC energy sources. DC microgrid is smaller in installation size and more cost effective as compared to AC microgrid. It requires a lesser number of power electronic converters; hence, the overall efficiency is improved. In addition, $\mathrm{AC} / \mathrm{DC}$ converters do not require a transformer, which reduces the size of the DC microgrid significantly. An increasing use of DC microgrids is also in trains, ships, charging facilities for electric vehicles [5]. The occurrence of faults in the physical and communication network layer in the power system can create an imbalance in the overall system. Hence, to maintain the reliability and stability of the overall power system, fault detection forms an important aspect of the overall monitoring systems. For protecting the DC microgrid system from different types of faults, fault detection techniques [6,7] are studied and advanced protection schemes are implemented. The possible faults in a DC microgrid can be categorized as actuator fault, sensor fault, component fault and interconnection fault between the subsystems. The detection of these faults in multiple interconnceted DGUs [8] are explained. 
The increased use of sensor network software has drawn many research attentions recently. The quality of the collected data from the sensor readings plays an important role in driving the overall system uninterruptedly. Sensor faults are defined as a data-centric point of view. These faults can be caused by malfunction of hardware or software. The hardware sensor faults can occur due to damage of sensor, short circuits, low battery and errors due to calibration. The software faults can occur due to data logging process and results in abnormal data or short faults. Generally, sensor fault detection methods are categorized into four different types. They are a rule-based detection method, estimationbased method, time series analysis-based method and learning-based method [7]. The errors due to sensor faults should be detected immediately so that there is no loss of any network information and remedial actions are promptly taken. Most of the existing works on sensor fault detection work well with additive faults, but fail to work with multiplicative sensor faults. A validation of the sensor for a structural system with multiplicative sensor fault [9] is presented. Single sensor faults are more likely to occur in small scale systems, however, in interconnected systems like a DC microgrid, the occurrence of both voltage and current sensor faults should be considered along with actuator faults, interconnected line faults and component faults.

Sensor fault detections are discussed in grid connected or islanded operation mode using model-based and data driven techniques [10,11]. In the model-based fault diagnosis method [12], a state observer is designed to generate the residuals and a common threshold is selected. An alarm will be triggered when the generated residual exceeds the threshold [13]. In model-based fault detection, an identification method for switching power converters is designed using a model-based state estimator method. This type of approach is used to detect arbitrary faults in the sensor and components of the switching power converters in a nanogrid. The faults in the components, actuators and sensors of a DC microgrid are modeled by using additive terms in the state space and measurement equations. However, due to fluctuations in load and parameter uncertainties, the generated residuals may trigger a false alarm and accurate detection of fault will not be accomplished. Hence, to encounter this issue, different techniques like $H_{\infty} / H_{-}[12,14]$ criteria can be used for the design of observers. This criteria gives a balance trade off between the robustness of the residual and sensitivity from the faults to the generated residual in the presence of disturbance. This criteria can also be used as an attenuation of disturbance and thus enhance the robustness and stability of the system. Similarly, in [15], a bank of Linear Parameter Varying (LPV) observers are designed to detect, estimate and diagnose faults on every subsystem of the microgrid. The solutions of those observers are found by the $\mathrm{H}_{2} / \mathrm{H}_{\infty}$ sensitivity minimization problem which can guarantee robustness towards load disturbance and insensitivity to measurement noise. However, this criteria is mostly dependent on frequency domain aspects and provide less information about the stability condition of the observer. In [16], a wireless sensor network (WSN)-based wind turbine is considered for discussion of sensor fault detection and isolation. In [17], three types of communication delays and the states of the inverter in the microgrid are estimated using sliding mode estimation technique.

In [18], a linear observer based on Kalman Filter is designed to generate the sensor fault residuals and hence detect the faults. This method uses online recursive model estimation to detect a normal and a faulty sensor. However, the changes of load current in the power distribution decreases the robustness of the system performance. In [19], a sliding mode observer (SMO) for fault detection is designed based on the current and measured voltage of the capacitor in a modular multilevel converters. Adaptive SMObased fault tolerant control strategy is designed to reject the influence of faults in the pitch of a wind turbine in [20]. Similarly, in [21], multiple SMO is used for the estimation of state and unknown input for a class of MIMO nonlinear systems. In [22], a sensor fault was diagnosed in an electric traction pulse width modulated rectifier based on SMO. However, the SMO approaches based on first-order approaches suffer from the chattering effect caused due to measurement noise and unknown loads. Hence, to reduce the noise induced 
chattering, a higher order sliding mode observer (HOSM) $[23,24]$ based on Supertwisting Algorithm (STA) has been used. In [25], sensor faults are considered in a nonlinear aircraft model and estimated using a second order sliding mode observer and active fault tolerant control scheme is used. This observer can guarantee finite time convergence, reconstruct the unknown input and also provide robustness against bounded uncertainties. In [8], event triggered STA is used in sliding mode controller design for suppressing the matched and unmatched uncertainties in a grid connected and islanded mode microgrid. In [26], possible faults like actuator fault, sensor fault, component faults and interconnected lines fault are detected by using a distributed observer along with a hierarchical controller in a DC microgrid. Motivated by the aforementioned works, an HOSM observer is designed for sensor fault detection and attenuation of unknown loads in the DC microgrid.

In this proposed work, two HOSM observers based on STA are designed for every DGU to estimate current and voltage and generate the residuals for detection of faulty sensors. Two separate observers are designed for estimation of the current and voltage dynamics for each DGU. The selection of threshold plays an important role due to the interconnections of DGUs and for multi-fault occurrence scenarios. Multiple simulations are performed under on load and off load conditions with a multiplicative sensor fault for various DGU configurations and combinations of sensor faults. The changes in the generated residuals are observed and a common threshold for fault detection is finally identified for the DGUs. Simulations for various fault scenarios are conducted to validate the approach. The rest of the paper is organized as follows: The problem formulation is discussed in Section 2. Section 3 covers the HOSM observer design and Section 4 follows up with fault detection. Section 5 presents the simulation results whereas the discussion is provided in Section 6. Finally Section 7 concludes the paper.

\section{Problem Formulation}

The DC microgrid model with $N$ interconnected DGUs is shown in Figure 1. The DGUs in a DC microgrid are interconnected via power lines. The DGUs represent the nodes, $\mu=1, \ldots N$ and the power lines represent the edges, $\kappa_{e} \subseteq \mu \times \mu$ of a graph $G_{e}=\left(\mu, \kappa_{e}\right)$ which represent the microgrid. The interaction between the neighboring DGUs $i$ and $j$ can be represented by an adjacency matrix $T=\left[t_{i j}\right]$ where $t_{i j}$ is the possibility of interaction and $T$ represents the topology of the power system network. If there exists an interconnection between the DGUs $i$ and $j, t_{i j}=1$ otherwise $t_{i j}=0$. Applying Kirchoff's voltage and current law to the electrical scheme in the block diagram as shown in Figure 2, the dynamics of the $i$ th DGU can be represented as follows:

$$
\begin{gathered}
\frac{d V_{i}}{d t}=\frac{1}{C_{n i}}\left(I_{i}(t)-I_{l i}(t)\right)+\sum_{j \in N_{i}} \frac{1}{C_{n i} R_{i j}}\left(V_{j}(t)-V_{i}(t)\right) \\
\frac{d I_{i}}{d t}=\frac{1}{L_{n i}} V_{n i}(t)-\frac{R_{n i}}{L_{n i}} I_{i}(t)-\frac{1}{L_{n i}} V_{i}(t), i=1,2, \ldots \ldots N .
\end{gathered}
$$

where $V_{i}(t), I_{i}(t), C_{n i}, L_{n i}, R_{n i}, R_{i j}$ and $I_{l i}$ denote the load voltage, current generated, capacitor of the shunt, inductance, resistance, resistance of the line and overall current demand, respectively. The system states are represented by $V_{i}(t), I_{i}(t)$ and the input is represented by $V_{n i}(t)$. The point of common coupling (PCC) voltage of the DGU $i$ 's neighbors can be represented by $V_{j}(t)$ and $\frac{1}{R_{i j}}$ denotes the conductance of the power line connected to the DGU $i$ with its neighboring DGU. The DGU consists of a source voltage, which is a renewable resource, and the Buck converter acts as a supplier of local load connected to the PCC through a series of LC filters. The DC loads are unknown and can be treated as current disturbance $\left(I_{l i}\right)$. 


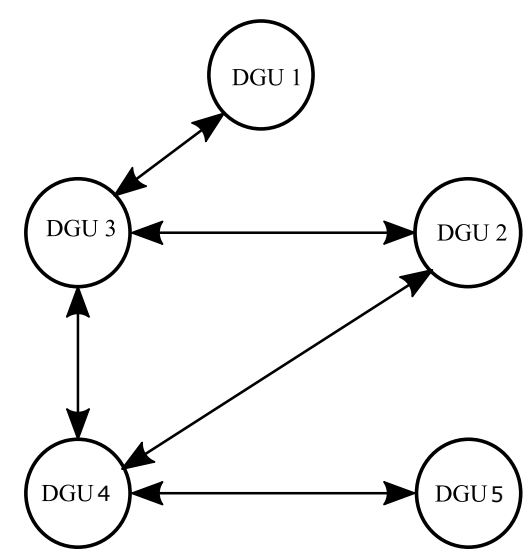

Figure 1. Communication network of 5 generation units (DGUs) in a DC microgrid.

The state space equations of the dynamics of each DGU can be represented as follows:

$$
\begin{gathered}
\dot{\chi}_{i}(t)=\Lambda_{i i} \chi_{i}(t)+\sum_{j \in N_{i}} \Lambda_{i j} \chi_{j}+\beta_{i} U_{i}(t)+Z_{i} \omega_{i}(t)+\beta_{d i} \zeta_{i}(t) \\
\psi_{i}(t)=\Gamma_{i} \chi_{i}(t)+\Delta_{d_{i}}(t) \zeta_{i}(t)
\end{gathered}
$$

where the local states can be represented by $\chi_{i}(t)=\left[V_{i}(t), I_{i}(t)\right]^{T}$, control input can be represented by $U_{i}(t)=V_{n i}(t)$, unknown input can be represented by $\omega_{i}(t)=I_{l i}(t)$, unknown disturbance can be represented by $\zeta_{i}(t)$ and the output by $\psi_{i}(t)$ with $\Gamma_{i}$ as the output matrix. The disturbance matrices can be represented by $\beta_{d i}$ and $\Delta_{d i}$. The DC microgrid state model can be represented as follows:

$$
\begin{aligned}
\Lambda_{i i}=\left[\begin{array}{cc}
\sum_{j \in N_{i}}-\frac{1}{R_{i j} C_{n i}} & \frac{1}{C_{n i}} \\
-\frac{1}{L_{n i}} & -\frac{R_{n i}}{L_{n i}}
\end{array}\right], \Lambda_{i j}=\left[\begin{array}{cc}
\frac{1}{R_{i j} C_{n i}} & 0 \\
0 & 0
\end{array}\right], \beta_{i}=\left[\begin{array}{c}
0 \\
\frac{1}{L_{n i}}
\end{array}\right], Z_{i}=\left[\begin{array}{c}
\frac{1}{C_{n i}} \\
0
\end{array}\right], \\
\beta_{d i}=\left[\begin{array}{c}
0.1 \\
0
\end{array}\right], \Delta_{d i}=\left[\begin{array}{c}
0.1 \\
0.1
\end{array}\right],
\end{aligned}
$$

A local primary controller is designed to ensure asymptotic stability for an augmented system. The primary controller, which is a decentralized sate feedback controller, regulates the voltage at each PCC and ensures that the microgrid is stable. An integrator term is used in the primary controller and its dynamics can be represented as follows:

$$
\dot{\epsilon}_{i}(t)=e_{i}(t)+\theta_{i}(t)=V_{r e f}(t)-V_{i}(t)+\theta_{i}(t)=V_{r e f}(t)-E_{i} \Gamma_{i} \chi_{i}(t)+\theta_{i}(t)
$$

where $V_{r e f_{i}}(t)$ is the reference voltage and $\theta_{i}(t) \epsilon \Re$ represents the secondary control input. The state feedback controller $U_{i}(t)$ can be represented as $U_{i}(t)=\varrho_{i} \chi_{i}^{\prime}(t)$, where $\chi_{i}^{\prime}(t)=$ $\left[\chi_{i}^{T}, \theta_{i}(t)\right]^{T}$ and it should ensure that $\left(\Lambda_{i i}^{\prime}+\beta_{i} K_{i}\right)$ is Hurwitz. The state matrix can be represented as $\Lambda_{i i}^{\prime}=\left[\begin{array}{cc}\Lambda_{i i} & 0 \\ -E_{i} & 0\end{array}\right]$ and $\varrho_{i}$ is a design parameter chosen using the Linear Matrix Inequality (LMI) conditions [27]. The DGUs can supply uninterrupted loads only when the proportional current sharing among the DGUs is ensured. Hence, at the steady state condition, the sharing of load current is proportionally done among all the other DGUs. Thus a secondary controller is designed for the $i$ th DGU as follows:

$$
\dot{\theta}_{i}(t)=-\Xi_{I, i} \sum_{j \in N_{i}} a_{i j}\left(\frac{I_{i}(t)}{I_{i}^{s}}-\frac{I_{j}(t)}{I_{j}^{s}}\right)
$$

where $\Xi$ represents the integral coefficient of the $i$ th DGU. The stability of the controller can be proved similar to [28]. 
The objective of the paper was to design an HOSM-based observer for current and voltage estimation for the system dynamics (1)-(2) for fault identification. Two separate observers are designed for estimation of the current and voltage dynamics for each DGU $i$ that will be discussed in the later sections. The selection of threshold plays an important role due to the interconnections of DGUs and multi-fault occurrence scenarios. In this paper, only a multiplicative sensor fault is considered for sensor fault detections. A hierarchical control scheme is used to maintain stability of the subsystem with an equal distribution of current among the DGUs. A decentralized primary controller is designed which guarantees the asymptotic stability of the connection between the DGUs. A secondary controller is also designed to ensure equal sharing of current between the DGUs and common value of average voltage is maintained in all the PCCs.

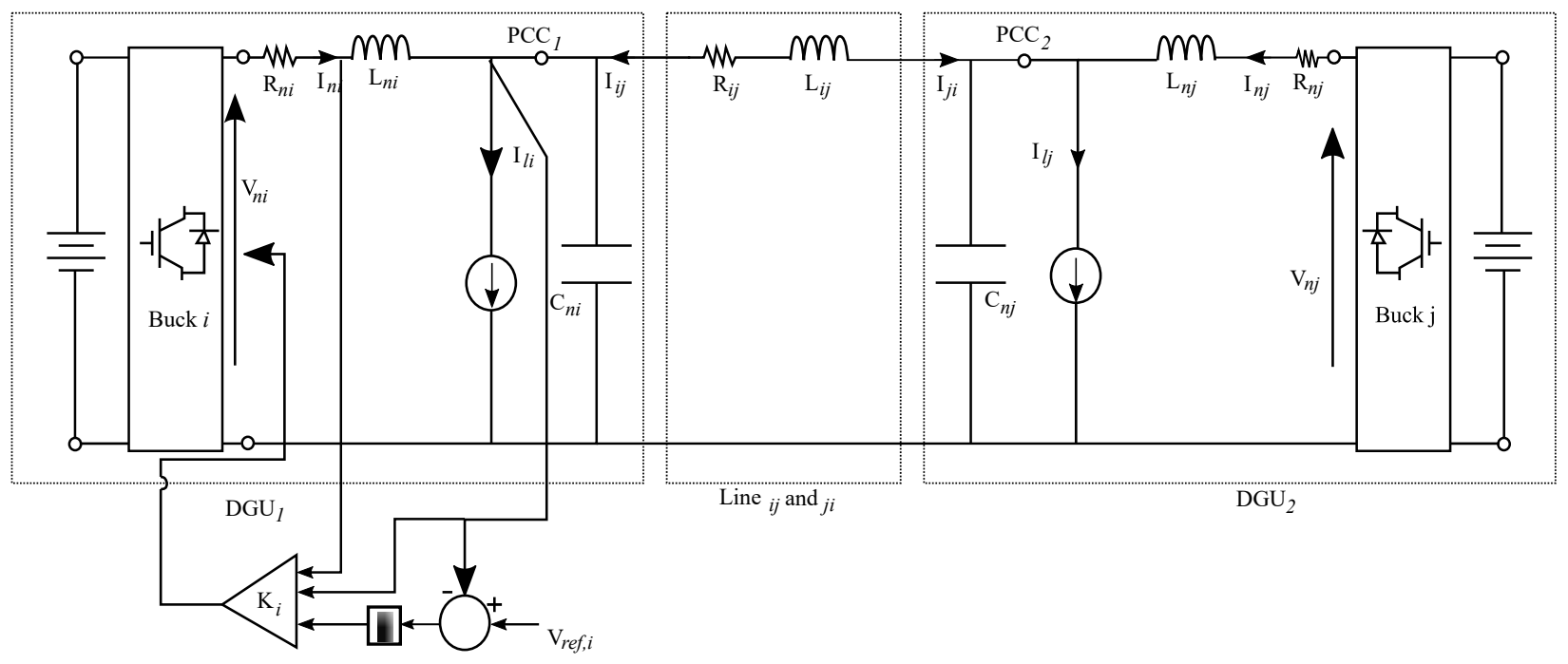

Figure 2. Electrical circuit model of two radially connected DGUs with a primary voltage controller.

\section{HOSM Observer Design and Fault Detection}

In this section, an HOSM observer [29] is designed to estimate the voltage and the current of the DGUs. The HOSM observer for voltage estimation can be designed by considering the current to be a known quantity and the voltage as an unknown quantity. The HOSM observer for estimating the unknown voltage from the current dynamics can be designed as follows:

$$
\left.\frac{d \hat{I}_{i}(t)}{d t}=\frac{1}{L_{n i}} V_{n i}(t)-\frac{R_{n i}}{L_{n i}} \hat{I}_{i}(t)-\frac{1}{L_{n i}} \varphi_{i}\left(s_{v i}\right)\right)
$$

where $\varphi_{i}(t), L_{n i}, R_{n i}, V_{n i}$, denote the corrective term of the super twisting (STA) [30], inductance and resistance of the power lines and control input to the Buck converter, respectively. The estimation error, $s_{v i}$, is defined as follows:

$$
s_{v i}(t)=\hat{I}_{i}(t)-I_{i}(t)
$$

where $\hat{I}_{i}$ and $I_{i}$ are the estimated and actual currents, respectively. The corrective terms of the STA can be designed according to [31], as

$$
\varphi_{i}\left(s_{v i}\right)=-K_{i 1}\left|s_{v i}\right|^{\frac{1}{2}} \operatorname{sign}\left(s_{v i}\right)-K_{i 2} \int_{0}^{t} \operatorname{sign}\left(s_{v i}\right) d \tau-K_{i 3} s_{v i}
$$


where $K_{i 1}, K_{i 2}$ and $K_{i 3}$ are the properly chosen positive gains. The sliding mode error dynamics can be represented in the following form:

$$
\dot{s}_{v i}=-\frac{R_{n i}}{L_{n i}} s_{v i}+\frac{1}{L_{n i}} \varphi_{i}\left(s_{v i}\right)+\frac{1}{L_{n i}} V_{i}=\lambda+\frac{1}{L_{n i}} \varphi_{i}\left(s_{v i}\right)
$$

where $\lambda$ represents the perturbation terms and can be expressed in the form $\lambda_{i}=\lambda_{i 1}\left(s_{v i}\right)+$ $\lambda_{i 2}$. Hence, the boundedness of the voltage can be assumed as $\left\|\lambda_{i 1}\right\| \leq \Delta_{i 1}|| s_{v i}||$ and $\left\|\dot{\lambda}_{i 2}\right\| \leq \Delta_{i 2}$, where $\Delta_{i 1}$ and $\Delta_{i 2}$ denotes the positive bounds of the perturbations. Based on the above boundedness conditions, the error convergence to zero can be proven to be asymptotic in finite time $t>T_{0}$ and the proof is similar to [31]. Thus, it can be seen that $I_{i}=\hat{I}_{i}$ in finite time. The finite time convergence of the sliding surface, i.e., $s_{v i}$ to the origin can be proved as shown in [30]. The positive gains $K_{i 1}, K_{i 2}$ and $K_{i 3}$ based on STA are designed as [32]. After the convergence of the sliding surface to the origin, the voltage of the $i$ th DGU can be reconstructed as:

$$
\hat{V}_{i}=K_{i 2} \int_{0}^{t} \operatorname{sign}\left(s_{v i}\right) d t
$$

The HOSM observer based on STA for the estimation of current can be designed as follows:

$$
\left.\frac{d \hat{V}_{i}(t)}{d t}=\frac{1}{C_{t i}}\left(I_{i}(t)-I_{l i}(t)\right)+\frac{1}{C_{t i} R_{i j}} V_{j}(t)-\frac{1}{C_{t i} R_{i j}} \varphi_{v i}\left(s_{c i}\right)\right)
$$

where $\varphi_{v i}\left(s_{c i}\right)$ is the corrective term of the STA. $C_{t i}, I_{i}, I_{l i}, R_{i j}$ and $V_{j}$ are the capacitance, current in the DGU, load current, resistance between the interconnected DGUs and voltage of the interconnected DGU, respectively. The current estimation error can be defined as $s_{c i}(t)=\hat{V}_{i}(t)-V_{i}(t)$. The robust HOSM terms are given as:

$$
\varphi_{v i}\left(s_{c i}\right)=-\kappa_{i 1}\left|s_{c i}\right|^{\frac{1}{2}} \operatorname{sign}\left(s_{c i}\right)-\kappa_{i 2} \int_{0}^{t} \operatorname{sign}\left(s_{c i}\right) d \tau-\kappa_{i 3} s_{c i}
$$

where $\kappa_{i 1}, \kappa_{i 2}$ and $\kappa_{i 3}$ are the positive chosen gains designed similar to the voltage observer. The estimation error dynamics can be written as:

$$
\dot{s}_{c i}=-\frac{1}{C_{t i}} s_{c i}-\frac{1}{C_{t i} R_{i j}} \varphi\left(s_{c i}\right)+\frac{1}{L_{n i}} V_{i}=\gamma_{i}+\frac{1}{C_{t i} R_{i j}} \omega\left(s_{c i}\right)
$$

The boundedness of current, $\gamma_{i}$, can be established with the positive constants $v_{i 1}$ and $v_{i 2}$ as $\left\|\gamma_{i 1}\right\| \leq v_{i 1}\left\|s_{c i}\right\|$ and $\left\|\dot{\gamma}_{i 2}\right\| \leq v_{i 2}$. Considering this condition, the finite time convergence of $s_{c i}$ can be ensured similar to [31]. Hence, the estimation of current can be achieved as:

$$
\hat{I}_{i}=\kappa_{i 2} \int_{0}^{t} \operatorname{sign}\left(s_{c i}\right) d t
$$

\section{Sensor Fault Detections}

The design of HOSM observers for fault detection is depicted in Figure 3. The multiplicative voltage and current sensor faults in the DGUs can be modeled as follows:

$$
\begin{aligned}
& \chi_{i, 1}^{\text {faulty }}(t)=V_{i}(t)\left(1+\lambda_{i}^{v}\right) \\
& \chi_{i, 2}^{\text {faulty }}(t)=I_{i}(t)\left(1+\lambda_{i}^{i}\right)
\end{aligned}
$$

where $-1<\lambda_{i}^{v}, \lambda_{i}^{i} \leq 0$. Based on this design, the voltage and current residuals can be selected to detect the faults immediately. At the instant of fault occurrence, the generated residual is compared with the selected thresholds. An alarm is set to trigger when a fault 
occurs in a system and then the residual signal exceeds the threshold. The residuals for fault detection, $R_{t h i}$, can be evaluated as follows:

$$
\begin{aligned}
R_{r_{i}}(t) & =\left|r_{i}(t)\right|, i=1,2 \ldots, N \\
R_{t h i} & =R_{r_{i}}(t)
\end{aligned}
$$

where $r_{i}=\hat{V}_{i}-V_{i}$ or $\hat{I}_{i}-I_{i}$ is the residual for voltage sensor fault and current sensor fault, respectively.

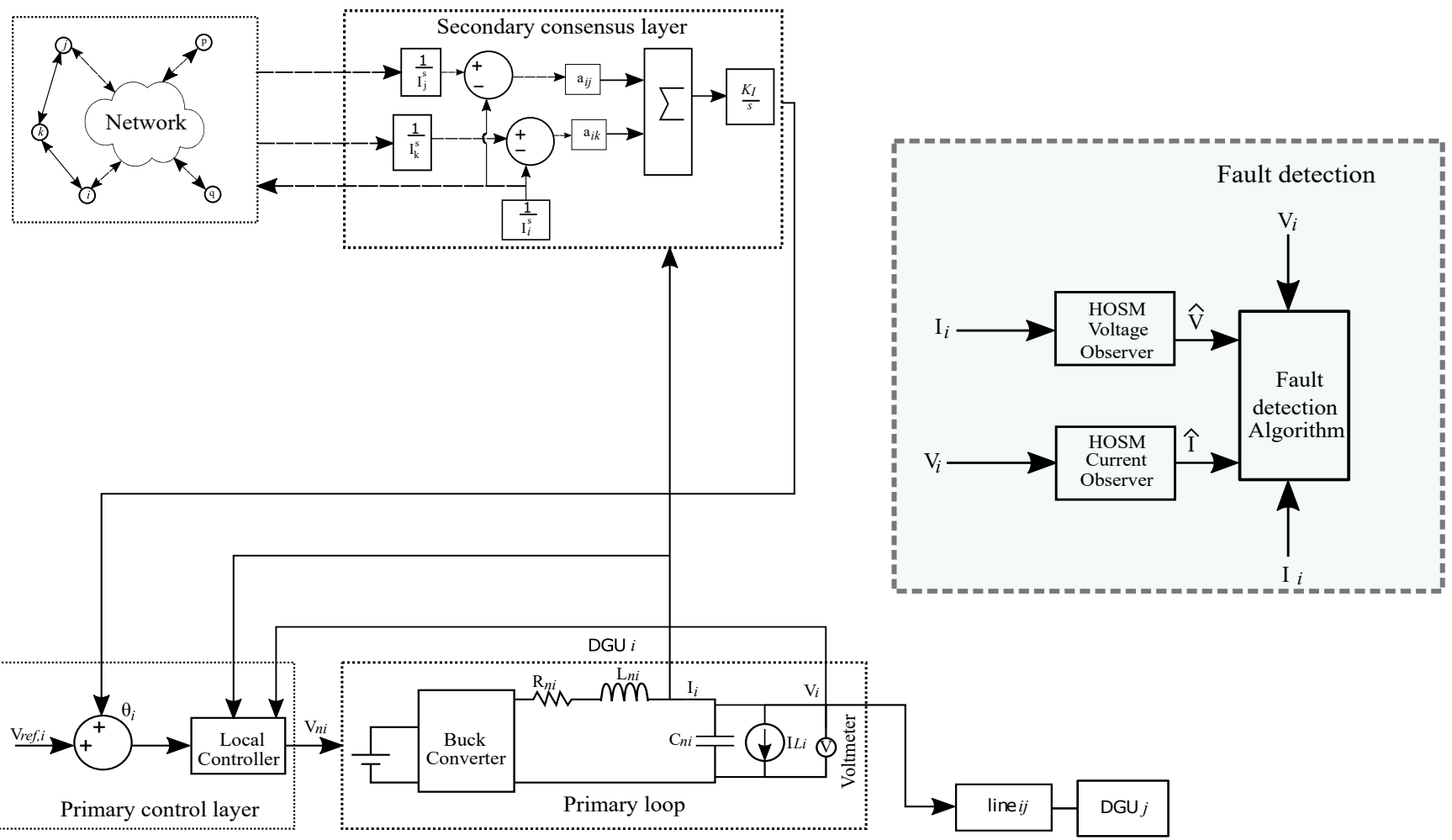

Figure 3. Functional block diagram of $i$ th DGU with controllers and higher order sliding mode (HOSM) observers.

\section{Simulation Results}

In this section, the simulation results are presented to demonstrate the efficiency and robustness of the HOSM observers for fault detection in a DC microgrid. The specification of the electrical and line parameters similar to [26] for DGUs are considered in Tables 1 and 2. A microgrid is comprised of 5 DGUs as shown in Figure 3. The HOSM observer based on Supertwisting algorithm (STA) is designed to generate the residuals of the faulty sensors. To check the robustness of the proposed methodology, a band limited white noise of $0.5 \mathrm{~dB}$ is considered for overall microgrid. The primary controller output of each DGU is shown in Figure 4 and the response of the HOSM observers for each DGU with healthy sensors is shown in Figure 5. Multiple cases of sensor faults are considered for analysis and graphically represented in this section.

Table 1. Electrical parameters of the DGUs.

\begin{tabular}{cccc}
\hline DGU & Resistance $\boldsymbol{R}_{\boldsymbol{n}}(\boldsymbol{\omega})$ & Capacitance $\boldsymbol{C}_{\boldsymbol{n}}(\mathbf{m F})$ & Inductance $L_{\boldsymbol{n}}(\mathbf{m H})$ \\
\hline DGU 1 & 0.2 & 2.2 & 1.8 \\
DGU 2 & 0.3 & 1.9 & 2.0 \\
DGU 3 & 0.1 & 1.7 & 2.2 \\
DGU 4 & 0.5 & 2.5 & 3.0 \\
DGU 5 & 0.4 & 2.0 & 1.3 \\
\hline
\end{tabular}


Table 2. Line parameters of the interconnected DGUs.

\begin{tabular}{ccc}
\hline Connected DGUs & Resistance $\boldsymbol{R}_{\boldsymbol{i j}}(\omega)$ & Inductance $L_{i j}(\mu \mathrm{H})$ \\
\hline$(1,3)$ & 0.07 & 2.1 \\
$(2,3)$ & 0.04 & 2.3 \\
$(2,4)$ & 0.08 & 1.8 \\
$(3,4)$ & 0.07 & 1 \\
$(4,5)$ & 0.05 & 2 \\
\hline
\end{tabular}

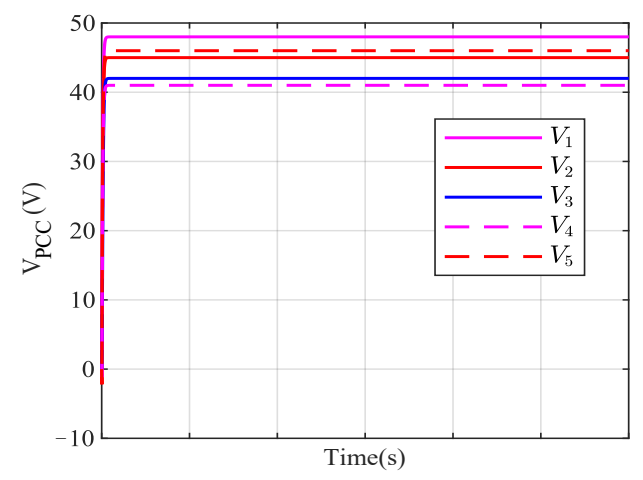

a) PCCs Voltage of the DGUs

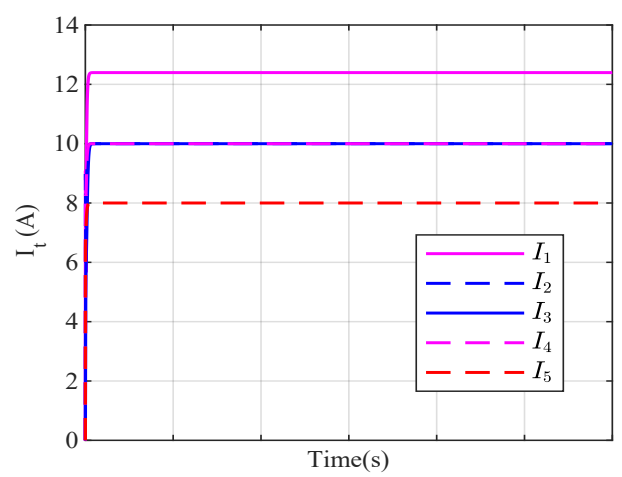

b) Output current of each DGUs

Figure 4. Primary controller output of each DGU. (a) PCCs Voltage of the DGUs; (b) Output current of each DGUs.

i) $\mathrm{DGU} 1$

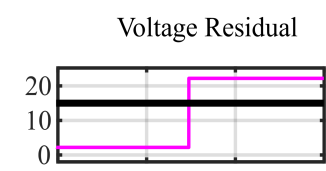

ii) DGU 2

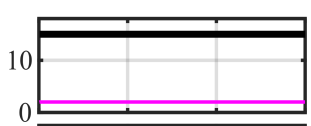

iii) $\mathrm{DGU} 3$

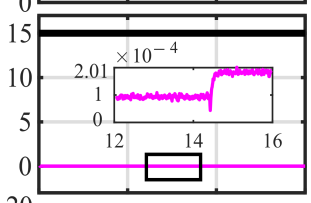

iv) $\mathrm{DGU} 4$

v) DGU 5

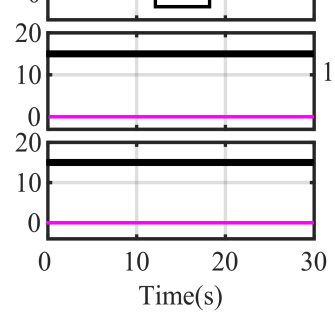

Current Residual
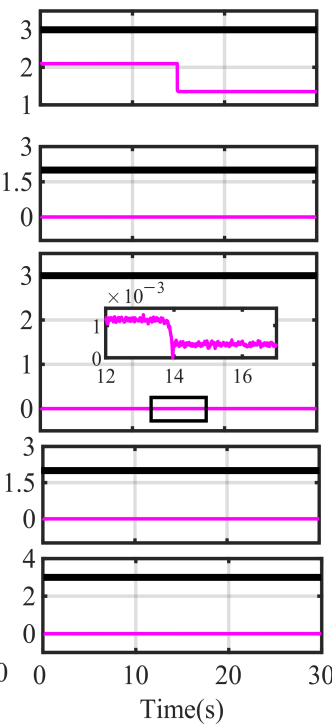

(a)

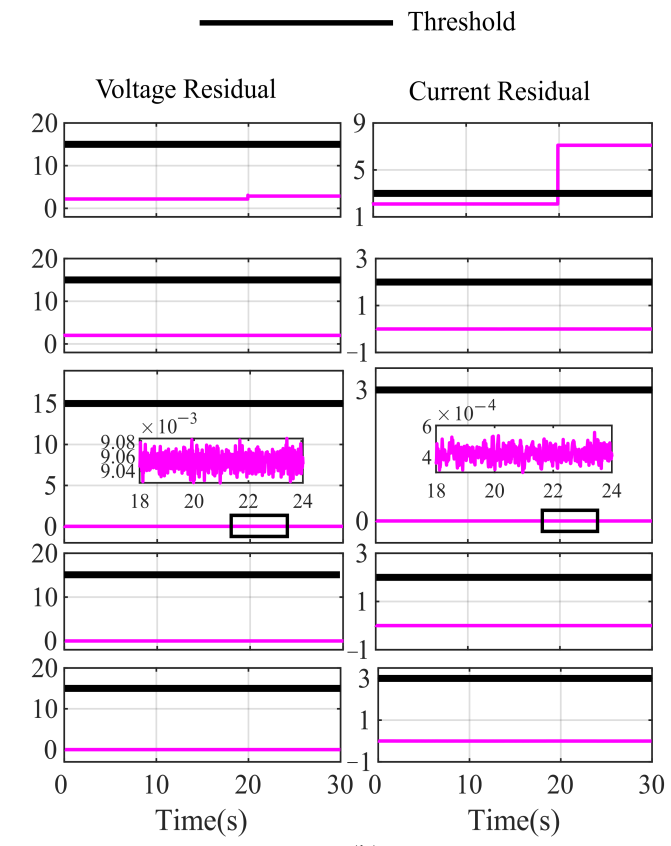

(b)

Figure 5. Performance of the observer during sensor faults in DGU 1. (a) Voltage and current residuals of the DGUs during voltage sensor fault; (b) Voltage and current residuals of the DGUs during current sensor fault.

\section{Threshold Selection}

In this subsection, the selection of the threshold for single and multiple sensor faults under different load conditions are evaluated. First, voltage sensor faults are considered for each DGU with an unknown load. Under this condition, the residuals of the estimated states and the faulty states are considered. Secondly, a current sensor fault is considered in all the DGUs at different time instants and a random noise of $1 \%$ to $3 \%$ is introduced in the 
faulty sensor. Due to current sensor fault in the DGUs, the voltage sensor also gets affected. The generated residuals are checked for all the current sensor faults. Thirdly, multiple sensor faults in a DGU and its interconnected DGUs are considered. The residuals of the faulty sensors are observed. After undergoing multiple simulations, the threshold for the voltage and current sensor faults are selected for accurate fault detection in a DC microgrid. Several rounds of simulations under different load, noise conditions are conducted to statistically validate the threshold values. After extensive analysis, the threshold values for the voltage and current sensor fault detections are identified as $15 \mathrm{~V}$ and $4 \mathrm{~A}$, respectively.

Case 1: Voltage sensor fault in a single DGU

In this case, a voltage sensor fault occurs in a single DGU and the response of all the DGUs are presented. As shown in Figure 5a(i), a voltage sensor fault occurs in DGU 1 at $\mathrm{t}=15 \mathrm{~s}$. Before fault, the voltage residuals lie below the threshold, however, after the occurrence of fault, the voltage residual in DGU 1, cross the threshold. A change in the residual of DGU 1 can be seen after $t=15 \mathrm{~s}$. As DGU 1 is interconnected to DGU 3, there is a change in residual of the respective voltage and current sensor. However, the voltage and current residuals of DGU 2, DGU 3, DGU 4 and DGU 5 lie below the threshold. Similarly, when there is a voltage and current sensor fault in DGU 2, DGU 3, DGU 4 and DGU 5, the interconnected DGUs also get effected. The residuals of the faulty sensors cross the threshold, however, the residuals of the non faulty sensor lie below the threshold. From these observations, the faulty sensor can be detected immediately and hence the overall system can be protected.

Case 2: Current sensor fault in a single DGU

In this case, a current sensor fault occurs in each DGU and the responses are observed. In Figure $5 b(i)$, a current sensor fault occurs in DGU 1 at $t=20 \mathrm{~s}$. The current residual cross the threshold after the occurrence of fault. At this instant, the fault can be detected using the fault detection algorithm. The effect of current sensor fault can be seen in the voltage sensor residual of DGU 1, however, it remains below the threshold. As DGU 1 is interconnected to DGU 3, the effect of the faulty current sensor can be seen in Figure 5b(iii). Similarly, when there is a current sensor fault in DGU 2, DGU 3, DGU 4 and DGU 5, the residuals of the faulty current sensors cross the threshold, indicating that a fault has occurred. Hence, the residual-based fault detection algorithm can differentiate a faulty sensor from a non faulty one.

Case 3: Voltage and current sensor faults

When both voltage and current sensor fault occurs in the DGUs of the microgrid, the respective voltage and the current sensor fault residuals cross the threshold and the flag raises at the instant of fault. The flag raises to 1 when a fault is detected and remains at 0 when there is no fault in the sensors. In Figure 6, a multiplicative voltage sensor fault $\lambda_{i}^{v}=-0.5$ occurs at $\mathrm{t}=15 \mathrm{~s}$ and for a current sensor $\lambda_{i}^{i}=-1$ occurs at $\mathrm{t}=20 \mathrm{~s}$. The voltage and current sensor faults are marked as Fault 1 and Fault , $_{2}$, respectively. At the instants of fault occurrence, the flags, Flag $v$ and Flag $i$ of the voltage and current sensor fault, respectively, raise to 1 as shown in Figure 6(ii). The changes in the interconnected DGU 3 are shown in Figure 6(iv). It can be seen that the residuals of the voltage and the current sensors in non-faulty DGUs lie below the threshold. In Figure $7 \mathrm{a}, \lambda_{i}^{v}=-0.9$ occurs at $\mathrm{t}=15 \mathrm{~s}$ and $\lambda_{i}^{i}=-1$ occurs at $\mathrm{t}=20 \mathrm{~s}$. Similarly, in Figure $8 \mathrm{a}$, the voltage and current sensor faults occur in the DGU 3 of the microgrid which is interconnected to DGUs 1, 2 and 4. The voltage fault in DGU 3 occurs at $t=10 \mathrm{~s}$ and the current fault occurs at $t=15 \mathrm{~s}$. The flag raises to 1 at that instants, indicating the occurrence of faults. The residual changes of the interconnected DGUs is portrayed in Figure 8(iii)-(v), respectively. Simulations are also done by considering $10 \%$ of load changes and introducing a random noise of $2 \%$ in the dynamics of the DC microgrid. A delay of $3 \mathrm{~ms}$ is also considered to check the robustness of HOSM observer-based sensor fault detection as shown in Figure 9. Faults are also considered in both the voltage sensors in DGU 1 and DGU 2 and the change in the residuals for the respective voltage and current sensor in all the DGUs is shown in Figure 10. 
i) DGU 1
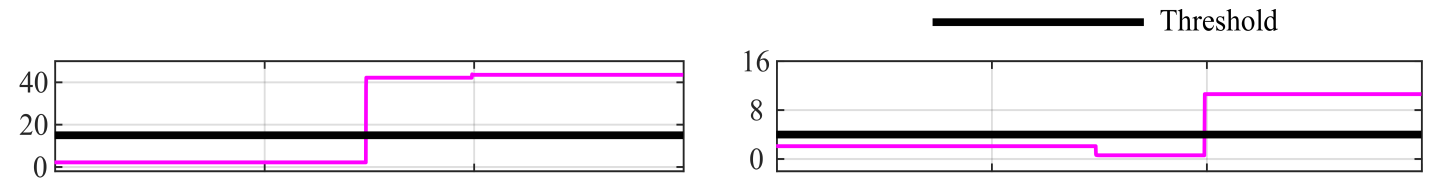

ii) Flag
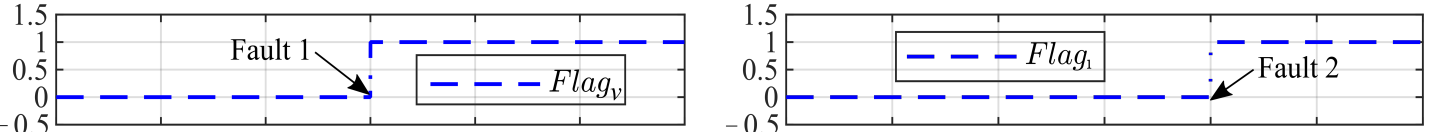

iii) DGU 2

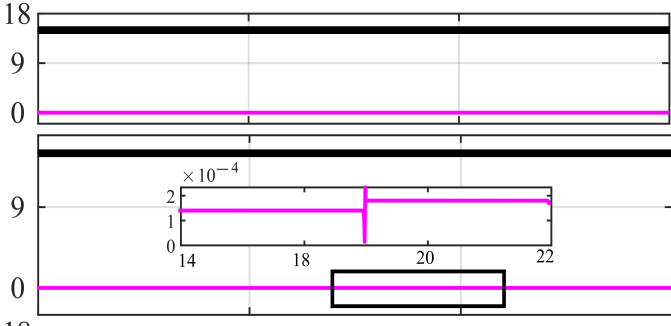

iv) DGU 3

v) DGU 4

vi) DGU 5

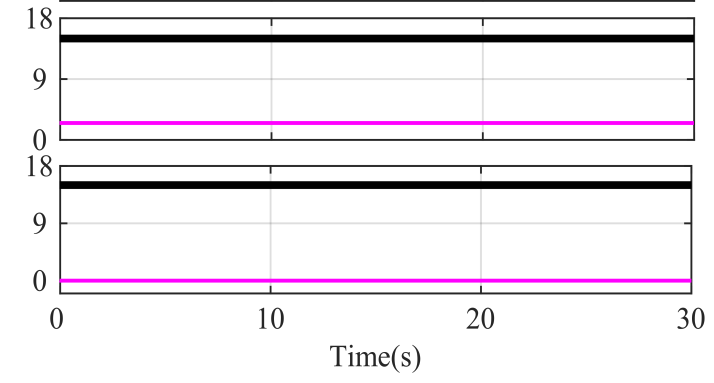

(a)
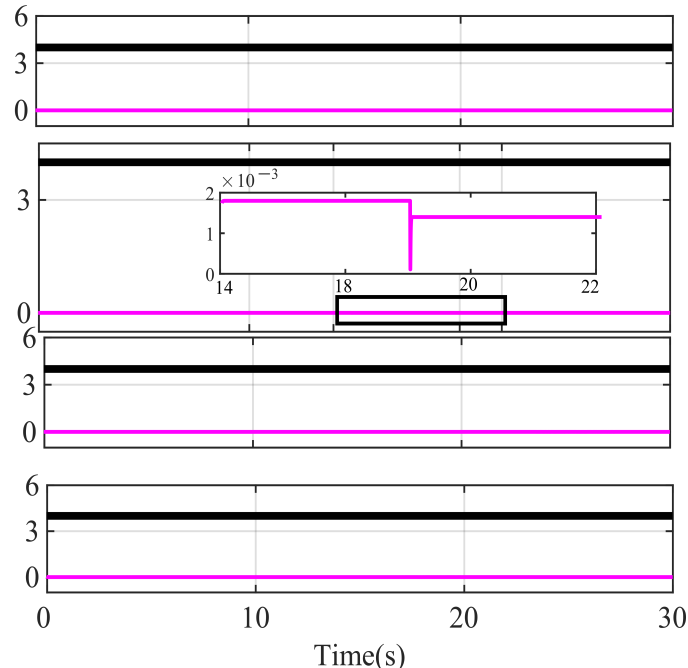

(b)

Figure 6. Residuals of the DGUs during voltage and current sensor fault in DGU 1 for $\lambda_{i}^{v}=-0.5$. (a) Voltage residuals of the DGUs; (b) Current residuals of the DGUs.

Threshold

i) DGU 1
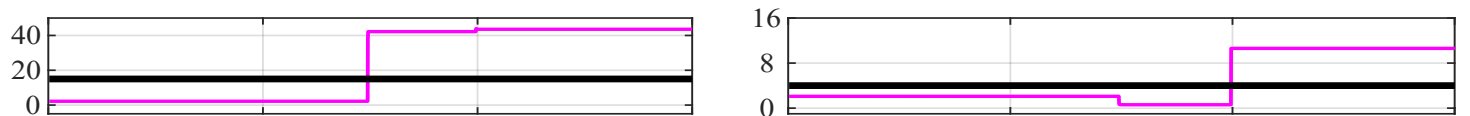

ii) Flag
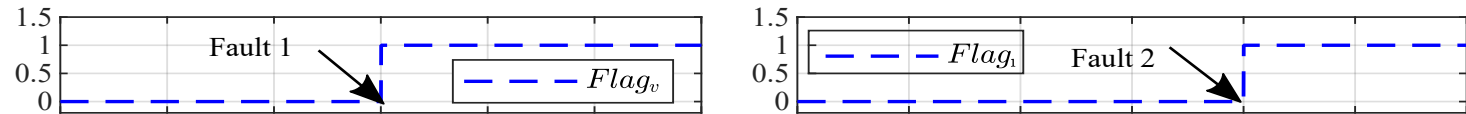

iii) $\mathrm{DGU} 2$
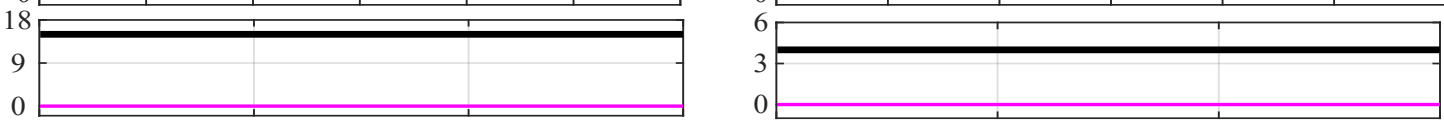

iv) $\mathrm{DGU} 3$

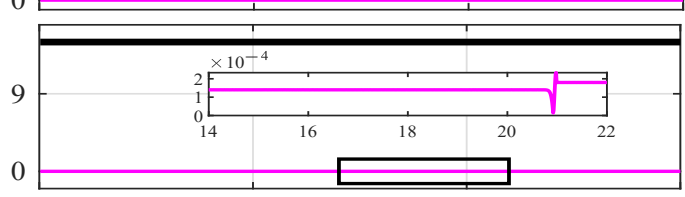

v) DGU 4

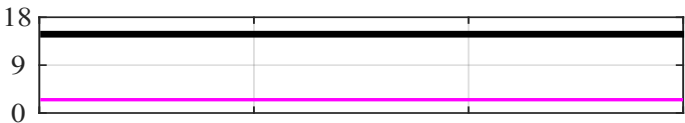

vi) DGU 5

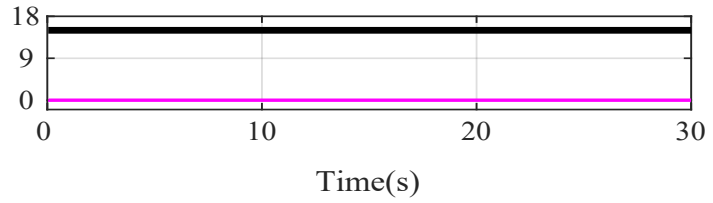

(a)
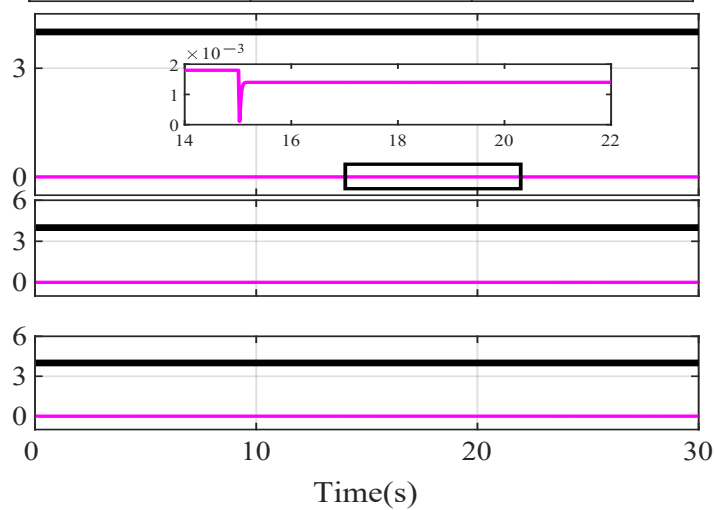

(b)

Figure 7. Residuals of the DGUs during voltage and current sensor fault in DGU 1 for $\lambda_{i}^{v}=-0.9$ and $\lambda_{i}^{i}=-1$. (a) Voltage residuals of the DGUs; (b) Current residuals of the DGUs. 
i) DGU 3
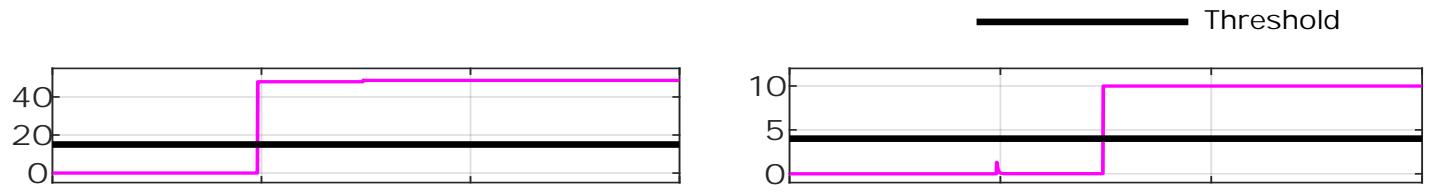

ii) Flag
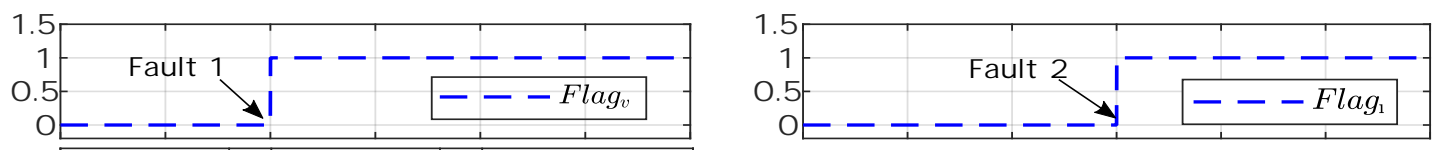

iii) DGU 1
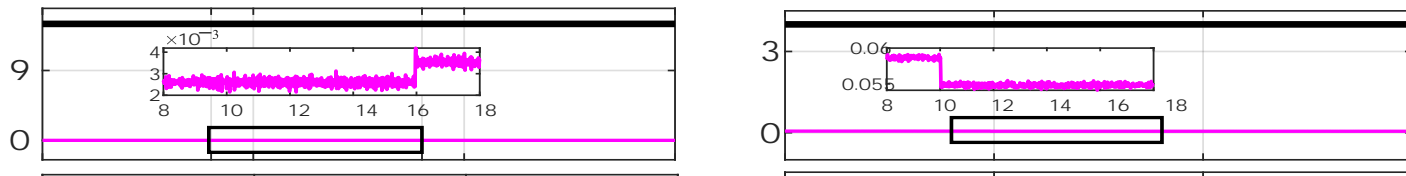

iv) DGU 2
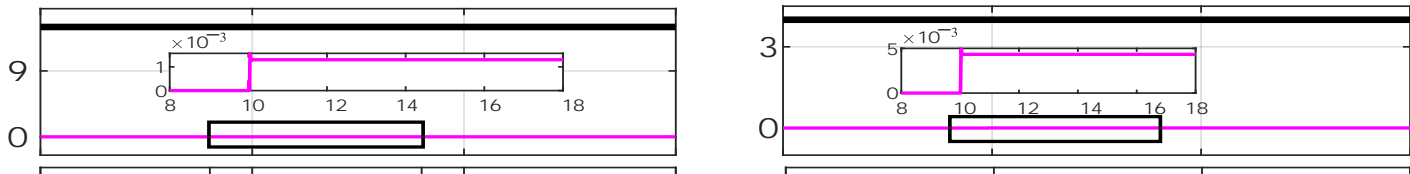

v) DGU 4

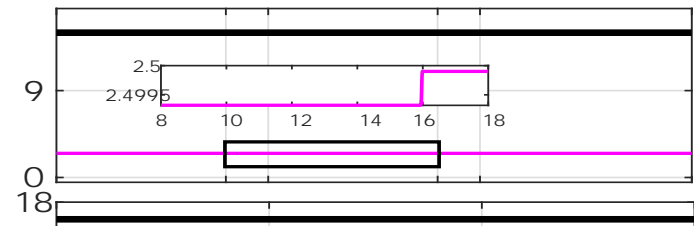

vi) DGU 5

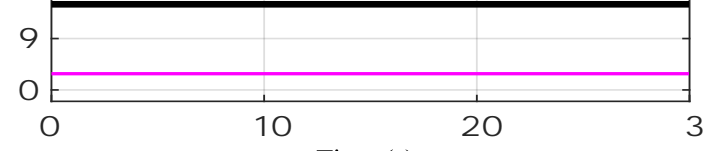

(a)

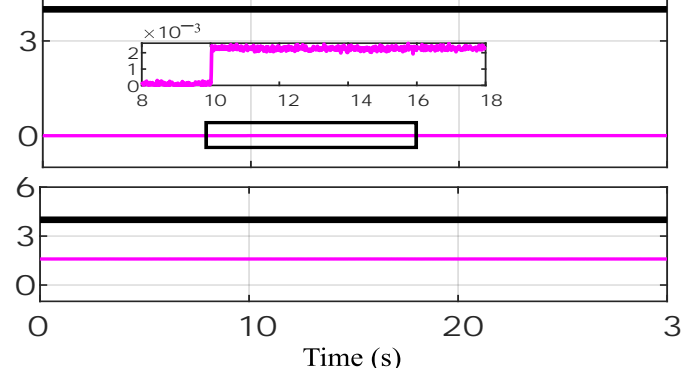

(b)

Figure 8. Residuals of the DGUs during voltage and current sensor fault in DGU 3. (a) Voltage residuals of the DGUs; (b) Current residuals of the DGUs.
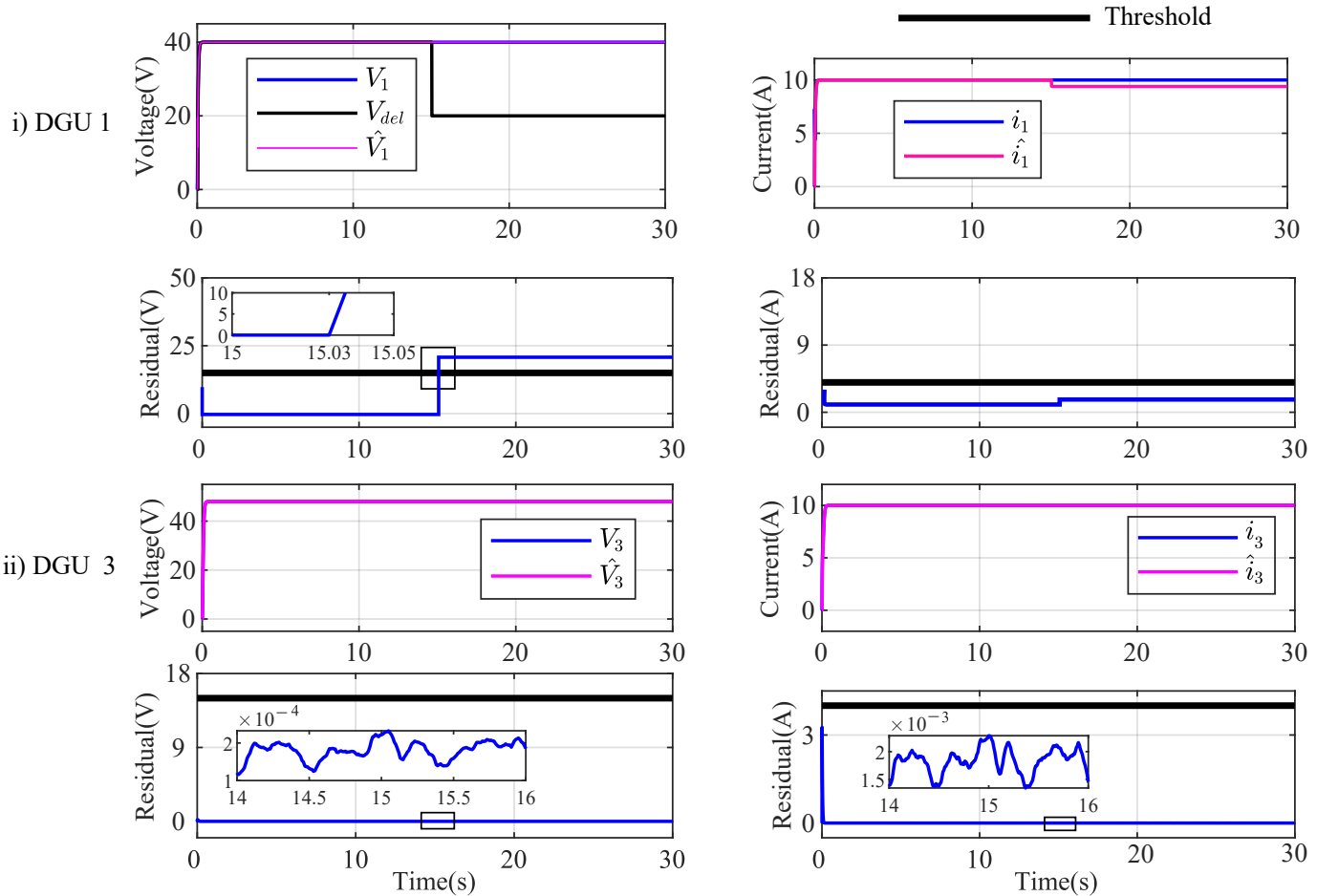

(a)

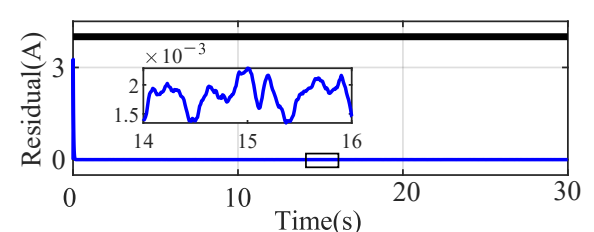

(b)

Figure 9. Response of the HOSM observer with delay and a voltage sensor fault in DGU 1 at $\mathrm{t}=15 \mathrm{~s}$. (a) Voltage residuals of the DGUs; (b) Current residuals of the DGUs. 
DGU 1

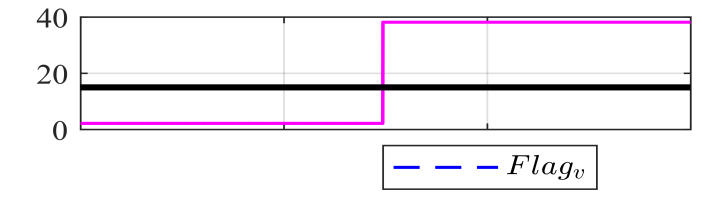

ii) Flag

iii)

DGU 2
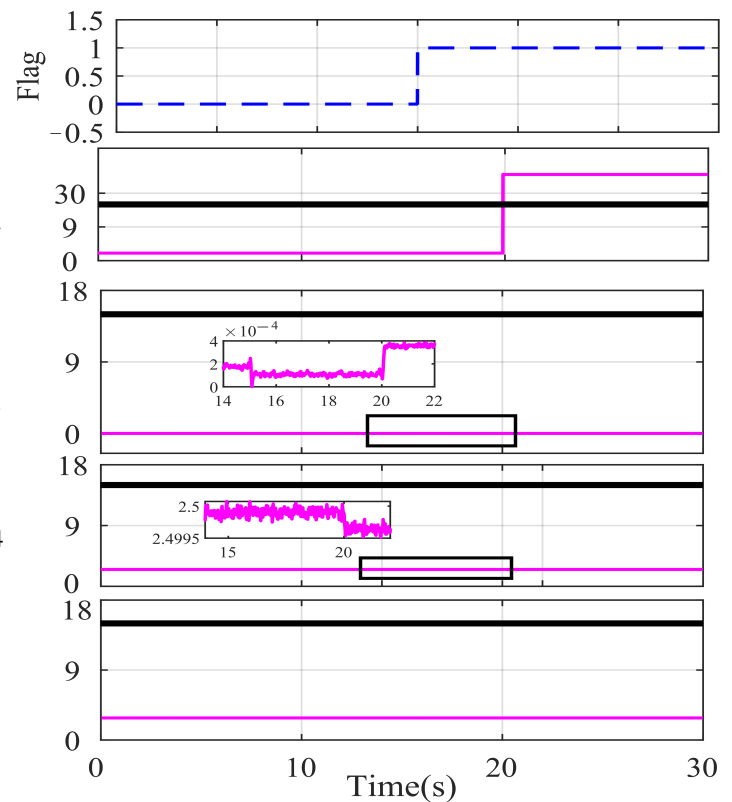

(a)

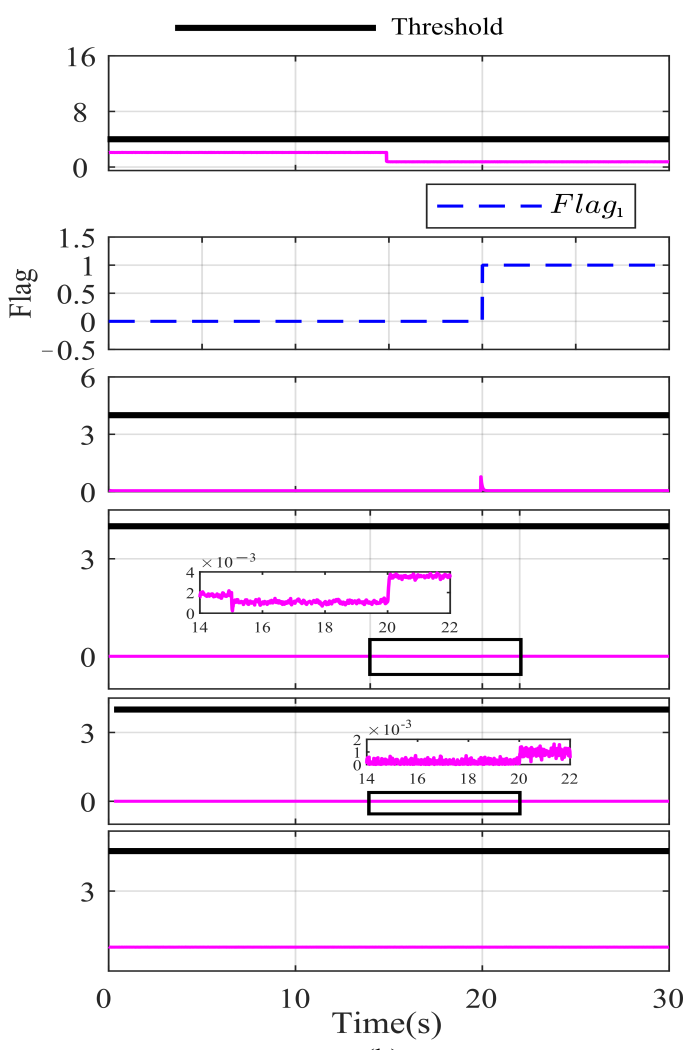

(b)

Figure 10. $(\mathbf{a}, \mathbf{b})$ Voltage residuals of the DGUs during voltage sensor fault in DGU 1 and DGU 2.

\section{Discussion}

The proposed method is analyzed with different sensor fault conditions and the results show that the proposed approach is successful in identification of single and multiple sensor faults in the interconnected DC microgrid system. In the voltage and current sensors, a multiplicative sensor faults with different values of $\lambda_{v}^{i}$ and $\lambda_{i}^{i}$ is considered in DGUs under various load conditions. A single sensor and multiple sensor fault scenarios are considered and multiple simulations are done to select a proper threshold for fault detection.

A random noise of $1-2 \%$ is considered along with the voltage and current sensor faults in all the DGUs and simulations are performed to check the residuals. It is seen that the residuals of the faulty sensors in the respective DGUs and its interconnected DGUs lie below the threshold. The optimal gain selection in HOSM significantly effects the residual-based fault detection performance of the DGUs. An incorrect choice of observer gains can raise the residual above the threshold thereby creating a false alarm of fault. To mitigate this issue of false alarm, the proper selection of observer gains is important. However, to check the robustness of the proposed methodology, a random noise of 1-3\% is introduced in DGU 1 and DGU 2. Optimal gain selection multiple voltage sensor faults occur in both the DGUs at $t=15 \mathrm{~s}$ and $\mathrm{t}=20 \mathrm{~s}$ as shown in Figure 10(iii). At the instant of faults, Fault $t_{v 1}$ and Fault $t_{v 2}$, the voltages in each residual cross the threshold. The impact of the voltage sensor faults of DGU 1 and DGU 2 in its common interconnected DGU 3 can be seen in Figure 10. The impact on the residuals of DGU 4 due to voltage sensor fault in DGU 2 is shown in the zoomed portion in Figure 10(iv). Thus, it can be ensured that the proposed method is robust to a noisy environment.

In this work, a constant delay between the Buck converter and the local controller is used to check the impact on the overall system. A delay of 3 samples equivalent to $0.03 \mathrm{~s}$ is considered in the voltage sensor of the DGU 1. It can be observed that the estimated voltage of the HOSM observer tries to track the original voltage even in the presence of delay. Similarly, the influence of the voltage sensor delay can be seen in the estimated 
current of DGU 1. As DGU 1 is interconnected to DGU 3 of the microgrid system, the estimated voltage and current tracks the actual voltage and current after a certain time delay. This proves the robustness of the HOSM observer. If the delay is increased then the system becomes unstable and the fault detection is further delayed. It can be seen that the voltage sensor fault is detected at $t=15.03 \mathrm{~s}$ for the three samples' delay as shown in Figure 9.

A change in $10 \%$ load in all the DGUs show a change in RMSE \% of $0.6 \%, 0.11 \%, 0.005 \%$, $0.0126 \%$ and $0.0288 \%$ in DGU 1-DGU 5 respectively from its nominal value. Similarly, the load changes of $15-20 \%$ are introduced in the DGUs and simulated. The HOSM observer is used to generate the residuals for faulty sensors. The faults with the said load conditions can be detected when the residuals cross the threshold. However, a change in $25 \%$ of the load causes a large deviation in the errors of the sensor estimations and the fault is not detected with the selected threshold. The RMSE values for 25\% load changes in DGU 1-DGU 5 are $1.3469 \%, 1.7508 \%, 1.9951 \%, 1.5871 \%$ and $1.7369 \%$. To test the robustness of the proposed method, $1 \%, 3 \%$ and $5 \%$ of the random noises are added to the actual measured signals. For the case of $5 \%$ noise, the proposed method was unable to detect the fault with the selected threshold. The proposed methodology is sensitive to higher sensor noise levels.

\section{Conclusions}

In this work, a sensor fault detection scheme is developed for interconnected DGUs of a DC microgrid. The two HOSM observers are designed for each DGU and the results show that the proposed method successfully detects the sensor faults in the voltage and current sensors. A sensor specific smart selection of threshold is done for the voltage and current sensors based on extensive simulation results. Results conclude that a multiplicative sensor fault can be detected by residual generation from an HOSM-based observer. The robustness of the proposed fault detection method is also established for various sensor faults varying conditions of delay, load and noise conditions. In the future, the proposed approach will also be extended to deal with actuator faults and delays.

Author Contributions: The contributions made by the authors are as follows: D.N.; Overall Design Framework, methodology and simulations: K.C.V.; Overall Supervision. All authors have read and agreed to the published version of the manuscript.

Funding: The research is supported by the National Research Foundation (NRF) of Korea through the Ministry of Education, Science and Technology under Grants NRF-2021R1A2C2012147.

Institutional Review Board Statement: Not applicable.

Informed Consent Statement: Not applicable.

Data Availability Statement: Not applicable.

Conflicts of Interest: The authors declare no conflicts of interest.

\section{References}

1. Tucci, M.; Meng, L.; Guerrero, J.M.; Ferrari-Trecate, G. Stable current sharing and voltage balancing in DC microgrids: A consensus-based secondary control layer. Automatica 2018, 95, 1-13. [CrossRef]

2. Kumar, D.; Zare, F.; Ghosh, A. DC microgrid technology: System architectures, AC grid interfaces, grounding schemes, power quality, communication networks, applications, and standardizations aspects. IEEE Access 2017, 5, 12230-12256. [CrossRef]

3. Salomonsson, D.; Sannino, A. Low-voltage DC distribution system for commercial power systems with sensitive electronic loads. IEEE Trans. Power Deliv. 2007, 22, 1620-1627. [CrossRef]

4. Yang, J.; Fletcher, J.E.; O'Reilly, J. Short-circuit and ground fault analyses and location in VSC-based DC network cables. IEEE Trans. Ind. Electron. 2011, 59, 3827-3837. [CrossRef]

5. Elsayed, A.T.; Mohamed, A.A.; Mohammed, O.A. DC microgrids and distribution systems: An overview. Electr. Power Syst. Res. 2015, 119, 407-417. [CrossRef]

6. Wang, T.; Liang, L.; Gurumurthy, S.K.; Ponci, F.; Monti, A.; Yang, Z.; De Doncker, R.W. Model-Based Fault Detection and Isolation in DC microgrids Using Optimal Observers. IEEE J. Emerg. Sel. Top. Power Electron. 2020. [CrossRef]

7. Sharma, A.B.; Golubchik, L.; Govindan, R. Sensor faults: Detection methods and prevalence in real-world datasets. ACM Trans. Sens. Networks (TOSN) 2010, 6, 1-39. [CrossRef] 
8. Kumar, V.; Mohanty, S.R.; Kumar, S. Event Trigger Super Twisting Sliding Mode Control for DC Micro Grid with Matched/Unmatched Disturbance Observer. IEEE Trans. Smart Grid 2020, 11, 3837-3849. [CrossRef]

9. Abdelghani, M.; Friswell, M.I. Sensor validation for structural systems with multiplicative sensor faults. Mech. Syst. Signal Process. 2007, 21, 270-279. [CrossRef]

10. Bansal, Y.; Sodhi, R. Microgrid fault detection methods: Reviews, issues and future trends. In Proceedings of the 2018 IEEE Innovative Smart Grid Technologies-Asia (ISGT Asia), Singapore, 22-25 May 2018; pp. 401-406. [CrossRef]

11. Hare, J.; Shi, X.; Gupta, S.; Bazzi, A. Fault diagnostics in smart micro-grids: A survey. Renew. Sustain. Energy Rev. 2016, 60, 1114-1124. [CrossRef]

12. Poon, J.; Jain, P.; Konstantakopoulos, I.C.; Spanos, C.; Panda, S.K.; Sanders, S.R. Model-based fault detection and identification for switching power converters. IEEE Trans. Power Electron. 2016, 32, 1419-1430. [CrossRef]

13. Gao, Z.; Cecati, C.; Ding, S.X. A survey of fault diagnosis and fault-tolerant techniques-Part I: Fault diagnosis with model-based and signal-based approaches. IEEE Trans. Ind. Electron. 2015, 62, 3757-3767. [CrossRef]

14. Liu, C.; Jiang, B.; Patton, R.J.; Zhang, K. Hierarchical-structure-based fault estimation and fault-tolerant control for multiagent systems. IEEE Trans. Control. Netw. Syst. 2018, 6, 586-597. [CrossRef]

15. Morato, M.M.; Regner, D.J.; Mendes, P.R.; Normey-Rico, J.E.; Bordons, C. Fault analysis, detection and estimation for a microgrid via H2/H LPV observers. Int. J. Electr. Power Energy Syst. 2019, 105, 823-845. [CrossRef]

16. Peng, Y.; Qiao, W.; Qu, L.; Wang, J. Sensor fault detection and isolation for a wireless sensor network-based remote wind turbine condition monitoring system. IEEE Trans. Ind. Appl. 2017, 54, 1072-1079. [CrossRef]

17. Yan, H.; Zhou, X.; Zhang, H.; Yang, F.; Wu, Z.G. A novel sliding mode estimation for microgrid control with communication time delays. IEEE Trans. Smart Grid 2017, 10, 1509-1520. [CrossRef]

18. Sardashti, A.; Ramezani, A.; Nezhad, H.S.; Moradmand, A. Observer-based Sensor Fault Detection in Islanded AC microgrids Using Online Recursive Estimation. In Proceedings of the 2019 6th International Conference on Control, Instrumentation and Automation (ICCIA), Sanandaj, Iran, 30-31 October 2019; pp. 1-6.

19. Shao, S.; Wheeler, P.W.; Clare, J.C.; Watson, A.J. Fault detection for modular multilevel converters based on sliding mode observer. IEEE Trans. Power Electron. 2013, 28, 4867-4872. [CrossRef]

20. Lan, J.; Patton, R.J.; Zhu, X. Fault-tolerant wind turbine pitch control using adaptive sliding mode estimation. Renew. Energy 2018, 116, 219-231. [CrossRef]

21. Veluvolu, K.; Soh, Y. Multiple sliding mode observers and unknown input estimations for Lipschitz nonlinear systems. Int. J. Robust Nonlinear Control 2011, 21, 1322-1340. [CrossRef]

22. Xia, J.; Guo, Y.; Dai, B.; Zhang, X. Sensor fault diagnosis and system reconfiguration approach for an electric traction PWM rectifier based on sliding mode observer. IEEE Trans. Ind. Appl. 2017, 53, 4768-4778. [CrossRef]

23. Kommuri, S.K.; Defoort, M.; Karimi, H.R.; Veluvolu, K.C. A robust observer-based sensor fault-tolerant control for PMSM in electric vehicles. IEEE Trans. Ind. Electron. 2016, 63, 7671-7681. [CrossRef]

24. Van Gorp, J.; Defoort, M.; Veluvolu, K.C.; Djemai, M. Hybrid sliding mode observer for switched linear systems with unknown inputs. J. Frankl. Inst. 2014, 351, 3987-4008. [CrossRef]

25. Chang, X.; Huang, J.; Lu, F. Sensor fault tolerant control for aircraft engines using sliding mode observer. Energies 2019, $12,4109$. [CrossRef]

26. Mola, M.; Afshar, A.; Meskin, N.; Karrari, M. Distributed Fast Fault Detection in DC microgrids. IEEE Syst. J. 2020. [CrossRef]

27. Boyd, S.; El Ghaoui, L.; Feron, E.; Balakrishnan, V. Linear Matrix Inequalities in System and Control Theory; SIAM: Philadelphia, PA, USA, 1994.

28. Tucci, M.; Riverso, S.; Vasquez, J.C.; Guerrero, J.M.; Ferrari-Trecate, G. Voltage control of DC islanded microgrids: A decentralized scalable approach. In Proceedings of the 2015 54th IEEE Conference on Decision and Control (CDC), Osaka, Japan, 15-18 December 2015; pp. 3149-3154.

29. Rinaldi, G.; Menon, P.P.; Edwards, C.; Ferrara, A. Higher Order Sliding Mode Observers in Power Grids with Traditional and Renewable Sources. IEEE Control Syst. Lett. 2019, 4, 223-228. [CrossRef]

30. Chalanga, A.; Kamal, S.; Fridman, L.M.; Bandyopadhyay, B.; Moreno, J.A. Implementation of super-twisting control: Supertwisting and higher order sliding-mode observer-based approaches. IEEE Trans. Ind. Electron. 2016, 63, 3677-3685. [CrossRef]

31. Moreno, J.A.; Osorio, M. Strict Lyapunov functions for the super-twisting algorithm. IEEE Trans. Autom. Control 2012, 57, 1035-1040. [CrossRef]

32. Rath, J.J.; Veluvolu, K.C.; Defoort, M.; Soh, Y.C. Higher-order sliding mode observer for estimation of tyre friction in ground vehicles. IET Control. Theory Appl. 2014, 8, 399-408. [CrossRef] 